\title{
Inhibitory Effects of Baicalein Derived from Japanese Traditional Herbal Medicine on SN-38 Glucuronidation
}

\author{
Takashi Satoh $^{l}$, Ayaka Igarashi ${ }^{2,3}$, Misaki Tanno $^{2,4}$, Koki Yamada $^{l}$, Natsuko Takahashi-Suzuki ${ }^{1}$, Kazuhiro Watanabe ${ }^{l}$ \\ ${ }^{I}$ Department of Pharmaceutics, Faculty of Pharmaceutical Sciences, Hokkaido University of Science \\ ${ }^{2}$ Department of Pharmaceutics, Hokkaido Pharmaceutical University School of Pharmacy \\ ${ }^{3}$ Department of Pharmacy, NTT East Japan Sapporo Hospital \\ ${ }^{4}$ Department of Pharmacy, Tomakomai Ohji General Hospital
}

Received, February 4, 2018; Revised, March 6, 2018; Accepted, May 3, 2018; Published, May 21, 2018.

\begin{abstract}
Purpose: The chemotherapeutic agent irinotecan is hydrolyzed to its active form SN-38 by human carboxyesterases, but SN-38 is converted into the inactive form SN-38G by hepatic UDPglucuronosyltransferases (UGTs). The aim of the present study was to evaluate the inhibitory effects of two $\beta$-glucuronidase-treated Japanese traditional herbal medicines (kampo), Hange-Shashin-To (TJ-14) and SaireiTo (TJ-114) on SN-38 glucuronidation, and the deglycosylation of baicalin (BG) and glycyrrhizic acid (GL) derived from TJ-14 and TJ-114 to form their respective aglycones, baicalein (BA) and glycyrrhetinic acid (GA). Methods: The inhibitory effects of $\beta$-glucuronidase-treated TJ-14 and TJ-114 on SN-38 glucuronidation by human liver microsomes were examined. BA and GA, which were enzymatically converted from BG and GL present in TJ-14 and TJ-114, were examined in the same manner. Furthermore, the enzymatic activities were measured by using recombinant UGT1A1 and UGT1A9 isoforms instead of human liver microsomes. BA, GA, SN-38, and their glycosides/glucuronides were analyzed with an LC-MS system. Results: As regards the linear initial reaction rate, $\mathrm{SN}-38$ glucuronidation by human liver microsomes was significantly inhibited by the addition of $\beta$-glucuronidase-untreated TJ-14 and TJ-114, but was more strongly inhibited by the addition of $\beta$-glucuronidase-treated TJ-14 and TJ-114. The results of LC-MS analysis and pharmacokinetic studies suggested that BA is the main inhibitor of SN-38 glucuronidation. In the Dixon plot, BA showed competitive inhibition of SN-38 glucuronidation, and the inhibition constant was $8.70 \pm 3.24$ $\mu \mathrm{M}$. Previous reports, studies of recombinant UGT isoforms indicated that SN-38 glucuronidation was mainly catalyzed by UGT1A1. Conclusions: These findings strongly suggested that SN-38 glucuronidation is inhibited by BA. BA could act as a pharmacokinetic regulating factor associated with SN-38 glucuronidation.

This article is open to POST-PUBLICATION REVIEW. Registered readers (see "For Readers") may comment by clicking on ABSTRACT on the issue's contents page.
\end{abstract}

\section{INTRODUCTION}

Irinotecan [ 7 - ethyl - 10 - [ 4 - ( 1 - piperidino $)$ - 1 - piperidino] carbonyloxy camptothecin (CPT-11) is a chemotherapeutic agent that exerts antitumor activity against several solid tumors. CPT-11 is predominantly hydrolyzed to a potent topoisomerase I inhibitor, 7 - ethyl - 10 - hydroxycamptothecin (SN38), by human carboxyesterases (1). The second metabolic pathway of CPT-11 is through cytochrome P450. CPT-11 is oxidized to 7 - ethyl - 10 [4 - N - (5 - aminopentanoic acid) - 1 - piperidino] carbonyloxy camptothecin (APC) and 7 - ethyl - 10 - (4 - amino 1 - piperidino) carbonyloxy camptothecin (NPC) by CYP3A4. Both APC and NPC are much less cytotoxic than CPT-11. However, carboxyesterase can hydrolyze NPC into SN-38 but not APC (2-4). The active form, $\mathrm{SN}-38$, is subsequently converted into the inactive form, 7 - ethyl - 10 hydroxycamptothecin glucuronide (SN-38G), by hepatic UDP - glucuronosyltransferases (UGTs) (5, $6)$.

Corresponding Author: Takashi Satoh; Department of Pharmaceutics, Faculty of Pharmaceutical Sciences, Hokkaido University of Science, 7-15-4-1 Maeda, Teine, Sapporo, Hokkaido, Japan, E-mail: tsatoh@hus.ac.jp

UGTs represent one of the major classes of enzymes and are involved in phase II conjugative metabolism. UGTs catalyze the transfer of a 
glucuronic acid moiety from uridine 5'diphosphoglucuronic acid (UDPGA) to a wide range of structurally diverse endogenous compounds (e.g., bile acid, bilirubin, and steroids) and xenobiotics (e.g., such drugs as $\mathrm{SN}-38$ and environmental toxicants). The resulting glucuronide conjugates are more polar than their parent compounds and are subsequently eliminated in bile or urine (5). Human UGTs are classified into four families: UGT1, UGT2, UGT3, and UGT8 (7). The most important drugconjugating UGTs belong to UGT1 and UGT2 families. Among the UGT1A isoforms, UGT1A1, $1 \mathrm{~A} 4,1 \mathrm{~A} 6$, and $1 \mathrm{~A} 9$ show moderate expression in the liver $(7,8)$. UGT1A1 is mainly responsible for the formation of SN-38G $(6,9,10)$.

The inactive form, $\mathrm{SN}-38 \mathrm{G}$, is excreted mainly into bile via such transporters as breast cancer resistance protein (BCRP), multidrug resistanceassociated protein (MRP), and P-glycoprotein (11, 12). $\mathrm{SN}-38 \mathrm{G}$ is deconjugated by $\beta$-glucuronidase present in intestinal microflora and regenerated to $\mathrm{SN}-38$. As a result, $\mathrm{SN}-38$ causes gastrointestinal toxicity by inducing mucosal damage. The major dose-limiting toxicity after administration of CPT-11 is severe myelosuppression (13) and late-onset diarrhea (14). In many cases, neutropenia caused by myelosuppression converges in a relatively short time and is treated with granulocyte colonystimulating factor (G-CSF) $(15,16)$. On the other hand, late-onset diarrhea continues to be a serious, debilitating, and life-threatening condition that leads to dehydration (17).

The Japanese traditional herbal medicine (kampo) Hange-Shashin-To (TJ-14) (17) and antibiotics, such as cefixime (18), are orally administered to ameliorate gastrointestinal toxicity because they inactivate bacterial $\beta$-glucuronidase, thereby suppressing the formation of toxic SN-38 from SN-38G (19). Kampo is prepared by combining multiple crude drugs and thus it contains many substances. As kampo is a combination of many substances, its effect is a combination of various interactions of the constituent substances (20). TJ-14 is commonly used to treat gastrointestinal disorders, such as acute and chronic gastrointestinal catarrh, fermentative diarrhea, and acute gastroenteritis. TJ14 is composed of seven medicinal herbs, one of which is Scutellariae radix (skullcap), the active ingredient of which is baicalin (BG). BG is the glucuronide of baicalein. A glucuronide is also a glycoside and the most specific term in the field. BG has one glucuronic acid molecule and is a natural glucuronide conjugate. The mechanism of action of
TJ-14 toward SN-38-induced late-onset diarrhea is considered to be bacterial $\beta$-glucuronidase inhibition by BG. As a result, BG alleviates SN-38-induced mucosal damage (21) and enzymatically hydrolyzed BA is absorbed by small intestinal epithelial cells through passive diffusion. TJ-14 also contains Glycyrrhizae radix (licorice). Glycyrrhizic acid (GL) is one of the active ingredients of licorice; it has two glucuronic acid molecules and is the glucuronide of glycyrrhetinic acid (GA). Orally administered GL is enzymatically hydrolyzed via 3monoglucuronyl-glycyrrhetinic acid (3MGA) to more bioactive GA by bacterial $\beta$-glucuronidase. Then, the deglycosylated forms, GA and BA, are absorbed into bloodstream and converted via 3MGA into GL and BG by hepatic UGTs $(22,23)$. On the other hand, SN-38 deconjugated by bacterial $\beta$ glucuronidase in intestine is taken up by the organic anion transporting polypeptide (OATP) 2B1 located at the apical membrane of enterocytes $(24,25)$ and absorbed into bloodstream. Based on the above findings, we hypothesized that BA and GA might influence hepatic UGT-mediated SN-38 glucuronidation. In this study, we examined whether the inhibitory effects of BA and GA originating from TJ-14 on UGT activity could be a pharmacokinetic regulating factor associated with $\mathrm{SN}-38$-induced late-onset diarrhea in vitro. In addition to TJ-14, another Japanese traditional herbal medicine, SaireiTo (TJ-114), which is composed of Scutellariae radix and Glycyrrhizae radix and used to treat severe diarrhea, was also examined.

\section{MATERIALS AND METHODS}

\section{Materials}

7-Ethyl-10-hydroxycamptothecin (SN-38) was purchased from Tokyo Chemical Industry (Tokyo, Japan). Glycyrrhetinic acid (GA) and 7-ethyl-10hydroxycamptothecin glucuronide (SN-38G) were purchased from Toronto Research Chemicals (Toronto, Ontario, Canada). Alamethicin, uridine 5'diphosphoglucuronic acid trisodium salt (UDPGA), and $\beta$-glucuronidase type HP-2 from Helix pomatia were purchased from Sigma-Aldrich (St. Louis, MO, U.S.A.). Pooled human liver microsomes BD Ultra Pool $^{\mathrm{TM}}$ HLM 150 (lot number 38291), recombinant UDP-glucuronosyltransferase (rUGT) $1 \mathrm{~A} 1$ and rUGT1A9 (Human UGT1A1 Supersomes ${ }^{\text {TM }}$ (lot number 4265007) and Human UGT1A9 Supersomes $^{\mathrm{TM}}$ (lot number 6307002)) were purchased from Corning Gentest (Woburn, MA, U.S.A.). Baicalin (BG) and glycyrrhizic acid (GL) 
were purchased from Wako Pure Chemical Industries (Osaka, Japan). Baicalein (BA) was purchased from Tocris Bioscience (Minneapolis, MN, U.S.A.). 3-Monoglucuronyl-glycyrrhetinic acid (3MGA) was purchased from Nacalai Tesque (Kyoto, Japan). Hange-Shashin-To (TJ-14) and Sairei-To (TJ-114) were obtained from Tsumura \& Co. (Tokyo, Japan). All other chemicals used in the experiments were of the highest purity commercially available.

\section{Composition of Japanese Traditional Herbal Medicine}

Japanese traditional herbal medicines TJ-14 and TJ114 were spray-dried hot-water extracts of a mixture of herbal crude drugs. According to the manufacturer's information, the total daily dose of $7.5 \mathrm{~g}$ of TJ-14 (three times a day) consists of $4.5 \mathrm{~g}$ of water-extracted herbal powder and $3.0 \mathrm{~g}$ of additives. The water-extracted powder $(4.5 \mathrm{~g})$ is prepared from a decoction of seven medicinal herbs: Pinelliae tuber 5.0 g; Scutellariae radix 2.5 g; Glycyrrhizae radix $2.5 \mathrm{~g}$; Zizyphi fructus $2.5 \mathrm{~g}$; Ginseng radix 2.5 g; Zingiberis siccatum rhizoma $2.5 \mathrm{~g}$; and Coptidis rhizome $1.0 \mathrm{~g}$. On the other hand, the total daily dose of $9.0 \mathrm{~g} \mathrm{TJ}-114$ (three times a day) consists of $6.0 \mathrm{~g}$ of water-extracted herbal powder and $3.0 \mathrm{~g}$ of additives. The water-extracted powder $(6.0 \mathrm{~g})$ is prepared from a decoction of twelve medicinal herbs: Bupleuri radix, $7.0 \mathrm{~g}$; Pinelliae tuber, $5.0 \mathrm{~g}$; Alismatis rhizome, $5.0 \mathrm{~g}$; Scutellariae radix, $3.0 \mathrm{~g}$; Ginseng radix, 3.0 g; Zizyphi fructus, 3.0 g; Poria, 3.0 g; Polyporus, 3.0 g; Atractylodis lanceae rhizome, 3.0 g; Cinnamomi cortex, $2.0 \mathrm{~g}$; Glycyrrhizae radix, $2.0 \mathrm{~g}$; and Zingiberis rhizome, $1.0 \mathrm{~g}$. Generally, kampo including TJ-14 and TJ-114 is taken with half a glass $(50-100 \mathrm{~mL})$ of water or warm water.

\section{$\beta$-Glucuronidase Treatment of Japanese Traditional Herbal Medicine}

The deglycosylation of BG to BA and GL via 3MGA to GA in TJ-14 and TJ-114 was conducted according to a previous report (26) with minor modifications. Ground TJ-14 and TJ-114 were added to $50 \mathrm{mM}$ sodium acetate buffer $(\mathrm{pH} 5.0)$. The $5.0 \mathrm{w} / \mathrm{v} \%$ suspensions were incubated without or with 200 units $/ \mathrm{mL} \beta$-glucuronidase for $8 \mathrm{hr}$ at $37^{\circ} \mathrm{C}$. A portion of the reaction solutions were passed through a solidphase extraction cartridge (Sep-Pak C18 Plus Short Cartridge, Waters, Milford, MA, U.SA.) and elution was carried out with methanol. The eluates were diluted to $0.5 \mathrm{w} / \mathrm{v} \%$ solutions using $50 \mathrm{vol} \%$ methanol and filtered through $0.45 \mu \mathrm{m}$ membrane filter. The filtrate diluted to one-tenth its original concentration was injected into a liquid chromatograph-mass spectrometer (LC-MS) system.

\section{Glucuronidation of $\mathbf{S N - 3 8}$}

The inhibitory effects of $\beta$-glucuronidase-treated TJ14 and TJ-114 on SN-38 glucuronidation in human liver microsomes were examined. Ten $\mu \mathrm{L}$ of a 0.5 $\mathrm{w} / \mathrm{v} \%$ solution of $\beta$-glucuronidase-treated or untreated TJ-14 or TJ-114 was added into a microtube and the solution was evaporated under reduced pressure to give a residue. The residue was added to an incubation mixture composed of $50 \mathrm{mM}$ Tris- $\mathrm{HCl}$ buffer ( $\mathrm{pH}$ 7.4) containing $10 \mathrm{mM} \mathrm{MgCl}_{2}$, $0.2 \mathrm{mg}$ protein $/ \mathrm{mL}$ human liver microsomes, and 25 $\mu \mathrm{g} / \mathrm{mL}$ alamethicin, and the mixture was kept on ice for $10 \mathrm{~min}$. Pre-incubation was carried out after the addition of SN-38 methanolic solution for $5 \mathrm{~min}$ at $37{ }^{\circ} \mathrm{C}$. The final concentration of methanol was adjusted to $1.0 \mathrm{vol} \%$. The reaction was initiated by adding $5 \mathrm{mM}$ UDPGA in a final volume of $100 \mu \mathrm{L}$, followed by incubation for $0-60 \mathrm{~min}$ at $37^{\circ} \mathrm{C}$. The reaction was terminated by adding $100 \mu \mathrm{L}$ of icecold acetonitrile to the reaction mixture. Precipitated proteins were removed by centrifugation for $5 \mathrm{~min}$ at 5,000 rpm and the supernatant was filtered through $0.45 \mu \mathrm{m}$ membrane filter. The filtrate was injected into a LC-MS system.

The inhibitory effects of BA and GA on SN-38 glucuronidation were examined in the same manner as described above except for the addition of $\beta$ glucuronidase-treated or untreated TJ-14 or TJ-114. Furthermore, except for the addition of rUGT1A1 or rUGT1A9 instead of human liver microsomes, the incubation was performed in the same manner as that described above.

\section{LC-MS System}

The mass chromatographs of BA, GA, SN-38, and their glycosides/glucuronides were measured with an LC-MS system (LCMS-2020 equipped with a model LC-20AD dual pump and an SPD-M20A UV detector at $254 \mathrm{~nm}$ ) with a reversed-phase column $(2.2 \mu \mathrm{m}$ particle size, $50 \times 2.0 \mathrm{~mm}$, i.d.; SHIMPACK XR-ODS (Shimadzu, Kyoto, Japan)) at the constant temperature of $40^{\circ} \mathrm{C}$. Chromatography was performed as follows.

The mobile phase consisted of solvents (A) methanol/water/formic acid (2:98:0.1, v/v/v) and (B) methanol/water/formic acid $(98: 2: 0.1, \mathrm{v} / \mathrm{v} / \mathrm{v})$. The gradient program for GL, 3MGA, and GA analysis was $0-3.5 \mathrm{~min}, 80-100 \% \mathrm{~B}$; $3.5-5.5 \mathrm{~min}, 100 \% \mathrm{~B}$; 
and $5.5-8.5 \mathrm{~min}, 80 \% \mathrm{~B}$ at the flow rate of 0.20 $\mathrm{mL} / \mathrm{min}$, and that for SN-38G, BG, SN-38, and BA analysis was 0-5.0 $\mathrm{min}, 40-62.5 \% \mathrm{~B}$; $5.0-7.5 \mathrm{~min}$, $62.5-100 \% \mathrm{~B}$; $7.5-9.5 \mathrm{~min}, 100 \% \mathrm{~B}$; and $9.5-12.5$ $\mathrm{min}, 40 \% \mathrm{~B}$ at the flow rate of $0.17 \mathrm{~mL} / \mathrm{min}$. The retention times of GL, 3MGA, GA, SN-38G, BG, SN-38, and BA were $1.4 \mathrm{~min}, 2.7 \mathrm{~min}, 4.2 \mathrm{~min}, 3.2$ min, $5.2 \mathrm{~min}, 6.7 \mathrm{~min}$, and $7.5 \mathrm{~min}$, respectively.

MS analysis was performed at the electrospray ionization interface with probe voltage set at $4.5 \mathrm{kV}$ and $-3.5 \mathrm{kV}$. Desolvation line and block heater temperatures were set at $250{ }^{\circ} \mathrm{C}$ and $400{ }^{\circ} \mathrm{C}$, respectively. Nitrogen was used as the nebulizing and drying gas at the flow rates of $1.5 \mathrm{~L} / \mathrm{min}$ and 15 $\mathrm{L} / \mathrm{min}$, respectively. Target ions (polarity) of GA, 3MGA, GL, BA, BG, SN-38, and SN-38G were set at $m / z 471.3(+), 647.4(+), 821.6(-), 268.9(-), 445.0$ $(-), 393.6(+)$, and $569.0(+)$, respectively. Chromatographic analysis was carried out on Shimadzu model 2020 data analysis software Lab Solutions Version 5.55.

\section{DATA ANALYSIS}

Kinetic parameters $\left(V_{\max }, K_{\mathrm{m}}\right.$, and $K_{\mathrm{si}}$ (substrate inhibition constant)) were estimated by fitting the Michaelis-Menten and substrate inhibition equations to the substrate concentrations and the initial reaction rates. Data analysis was performed with Enzyme Kinetics Module of SigmaPlot 13 (Systat Software, San Jose, CA, U.S.A.). The goodness of fit was evaluated on the basis of $\mathrm{R}^{2}$ values, AICc (Akaike's Information Criterion with adjustment for small sample size), and Sy.x (standard error of the regression). For the test of the difference between two independent groups, the Student's t test was conducted after the dispersion test by the $F$ test. The test of the difference between three independent groups was processed using the Dunnett method and the Tukey method after the dispersion test.

\section{RESULTS}

Time Course and Kinetic Profiles of SN-38 Glucuronidation in Human Liver Microsomes

In order to clarify the kinetic characterization of

Based on the above results, the concentration and the incubation time for SN-38 glucuronidation were set to $1.5 \mu \mathrm{M}$ and $20 \mathrm{~min}$ in the following experiments unless otherwise noted.
SN-38 glucuronidation, the effect of incubation time on SN-38 glucuronidation were examined. Human liver microsomes were incubated with $10 \mu \mathrm{M} \mathrm{SN}-38$ for $60 \mathrm{~min}$ (Figure 1). SN-38 glucuronidation activity increased in a time-dependent manner and the increase was linear up to $20 \mathrm{~min}$. Then, the effect of substrate concentration on $\mathrm{SN}-38$ glucuronidation in human liver microsomes was examined. Human liver microsomes were incubated with $0-100 \mu \mathrm{M}$ SN-38 for 20 min (Figure 2). SN-38 glucuronidation increased in a concentration-dependent manner up to $10 \mu \mathrm{M}$ and the increase was linear up to $2.0 \mu \mathrm{M}$ (inset in Figure 2). However, at concentrations higher than $20 \mu \mathrm{M}$, the kinetic profile of SN-38 glucuronidation indicated substrate inhibition. The enzyme kinetic parameters of $K_{\mathrm{m}}, V_{\mathrm{max}}, C L_{\mathrm{int}}$, and $K_{\mathrm{si}}$ were $9.0 \pm 0.79 \mu \mathrm{M}, 521 \pm 45.5 \mathrm{pmol} / \mathrm{mg}$ protein $/ \mathrm{min}, 58.3 \pm 3.3 \mu \mathrm{L} / \mathrm{mg}$ protein $/ \mathrm{min}$, and 48.5 $\pm 3.1 \mu \mathrm{M}$, respectively. The influence of TJ-14 and TJ-114 and their ingredients on SN-38 glucuronidation had to be examined under

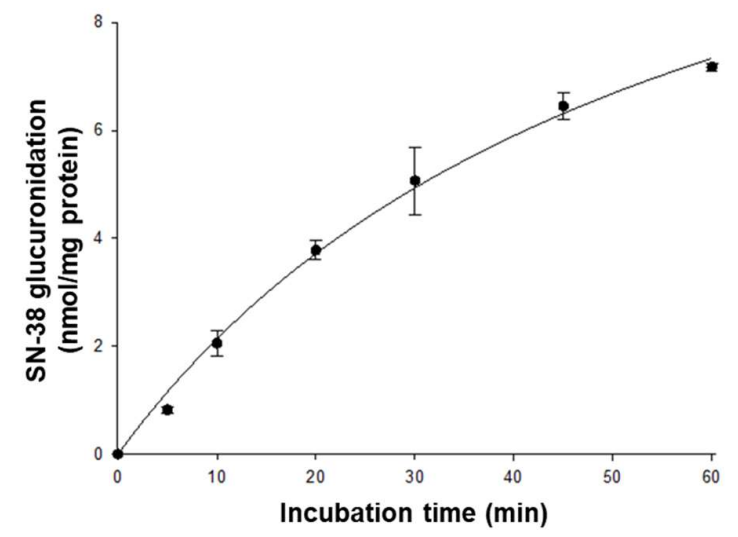

conditions that were not affected by substrate inhibition. From the kinetic parameters, $K_{\mathrm{si}} / K_{\mathrm{m}}$ ratio showed a high value of 5.4, and thus we used a concentration of SN-38 that was lower than the $K_{\mathrm{m}}$ value and range of the initial reaction rate period.

Figure 1. Effect of incubation time on SN-38 glucuronidation in human liver microsomes. SN-38 concentration was set to $10 \mu \mathrm{M}$. Each value represents the mean \pm S.D. of triplicate determinations. 


\section{Glucuronidation in Human Liver Microsomes}

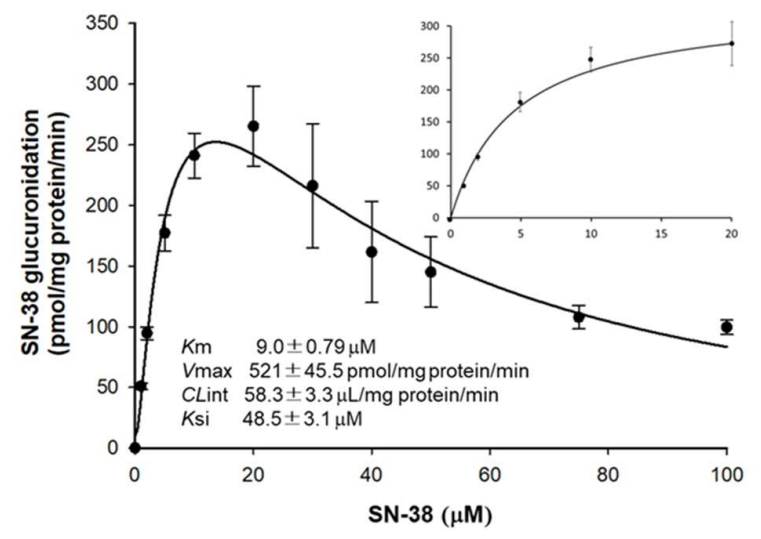

Figure 2. Kinetic profile and parameters of $\mathrm{SN}-38$ glucuronidation in human liver microsomes. The incubation was performed for $20 \mathrm{~min}$. Inset shows low concentration of SN-38. Each value represents the mean \pm S.D. of triplicate determinations.

\section{Inhibitory Effects of TJ-14 and TJ-114 on SN-38}

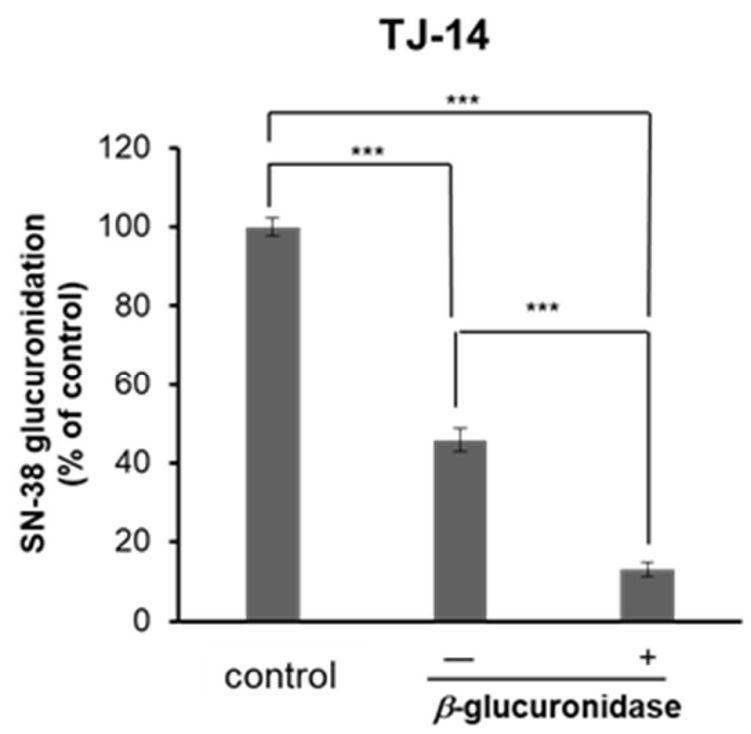

The inhibitory effects of $\beta$-glucuronidase-treated or untreated TJ-14 or TJ-114 on SN-38 glucuronidation were investigated. In Figure 3, the control activity of $\mathrm{SN}-38$ glucuronidation in human liver microsomes was $71.0 \pm 3.92 \mathrm{pmol} / \mathrm{mg}$ protein $/ \mathrm{min}$. Coincubation with untreated TJ-14 significantly decreased $\mathrm{SN}-38$ glucuronidation by $54 \%$ and coincubation with $\beta$-glucuronidase-treated $\mathrm{TJ}-14$ significantly reduced $\mathrm{SN}-38$ glucuronidation by $87 \%$. Similarly, co-incubation with untreated TJ-114 significantly decreased SN-38 glucuronidation by $48 \%$ and co-incubation with $\beta$-glucuronidase-treated TJ-114 significantly reduced $\mathrm{SN}-38$ glucuronidation by $72 \%$.

Table 1 shows the contents of five active ingredients $\mathrm{BG}, \mathrm{BA}, \mathrm{GL}, 3 \mathrm{MGA}$, and $\mathrm{GA}$ in $\beta$ glucuronidase-treated or untreated TJ-14 and TJ-114. The results indicated that deglycosylation by $\beta$ glucuronidase markedly increased BA obtained from BG but slightly increased GA from GL. Accordingly, it was considered that $\mathrm{SN}-38$ glucuronidation is strongly inhibited by aglycone BA rather than glycosides BG and GL, as shown in Figure 3.

Figure 3. Inhibitory effects of $\beta$-glucuronidase-treated or untreated TJ-14 or TJ-114 on SN-38 glucuronidation in human liver microsomes. SN-38 concentration was set to $1.5 \mu \mathrm{M}$ and incubation was performed for 20 min. Control activity was $71.0 \pm 3.92 \mathrm{pmol} / \mathrm{mg}$ protein $/ \mathrm{min}$. The final concentrations of TJ-14 and TJ-114 in the incubation mixture were set at $0.05 \mathrm{w} / \mathrm{v} \%$. Each value represents the mean \pm S.D. of triplicate determinations. Significantly different from control, $p<0.001$. 
Table 1. Contents of active ingredients derived from $\beta$-glucuronidase-treated or untreated TJ-14 and TJ-114

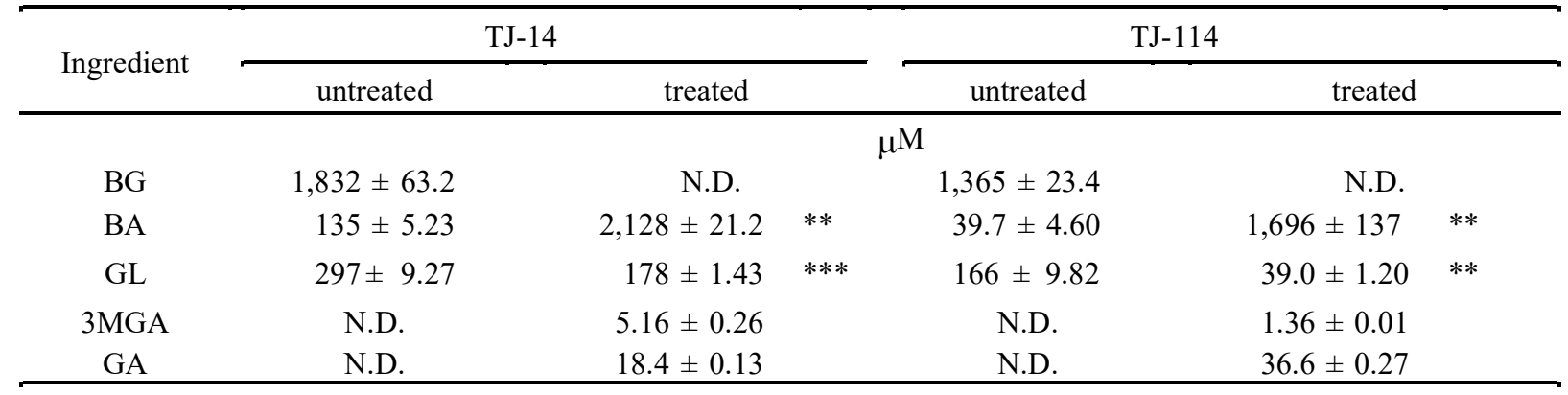

The $5.0 \mathrm{w} / \mathrm{v} \%$ suspensions were incubated without or with $200 \mathrm{units} / \mathrm{mL} \beta$-glucuronidase for $8 \mathrm{hr}$ at $37{ }^{\circ} \mathrm{C}$. Each value represents the mean \pm S.D. of triplicate determinations. Significantly different from control, ${ }^{* *} p<0.01, \quad p<0.001$. N.D.: less than $20 \mathrm{fmol}, 40 \mathrm{fmol}, 10 \mathrm{fmol}, 2.5 \mathrm{fmol}$, and $1.3 \mathrm{fmol}$ for BG, BA, GL, 3MGA, and GA, respectively.
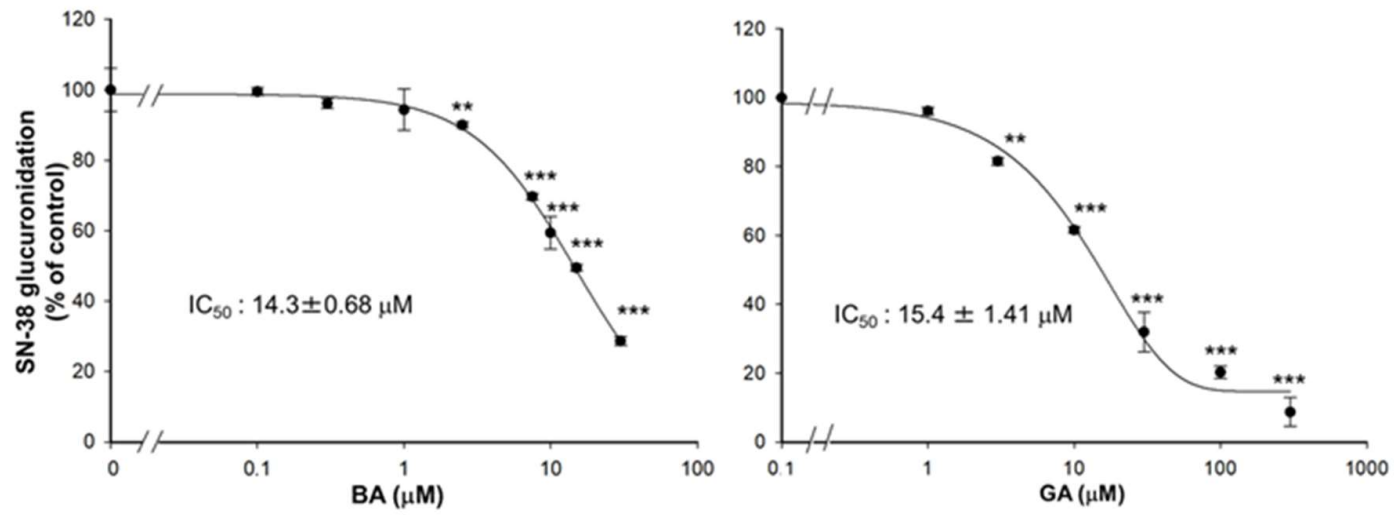

Figure 4. Concentration-dependent inhibition curves of BA and GA on SN-38 glucuronidation in human liver microsomes. SN-38 was set to $1.5 \mu \mathrm{M}$ and incubation was performed for $20_{* *}$ min. Each value represents the mean \pm S.D. of triplicate determinations. Significantly different from control, $\quad p<0.01, \quad p<0.001$.

Inhibitory Effects of $\mathrm{BA}$ and $\mathrm{GA}$ on $\mathrm{SN}-38$ Glucuronidation in Human Liver Microsomes The inhibitory effects of BA and GA on SN-38 glucuronidation in human liver microsomes were investigated. The concentration-dependent inhibition curves are shown in Figure 4. Coincubation with more than $3.0 \mu \mathrm{M}$ BA or GA significantly decreased SN-38 glucuronidation. Fifty percent inhibitory concentration $\left(\mathrm{IC}_{50}\right)$ values of $\mathrm{BA}$ and GA were $14.3 \pm 0.68 \mu \mathrm{M}$ and $15.4 \pm 1.41 \mu \mathrm{M}$, respectively.

Then, the Dixon plots were analyzed to clarify the types of inhibition and the inhibition constant $\left(K_{\mathrm{i}}\right)$ of BA in human liver microsomes. In Figure 5, the concentrations of SN-38 were set at $0.5,0.75$ (lower than $K_{\mathrm{m}}$ value), and $1.5 \mu \mathrm{M}$ ( $K_{\mathrm{m}}$ value). BA showed competitive inhibition of $\mathrm{SN}-38$ glucuronidation and the corresponding $K_{\mathrm{i}}$ value was $8.70 \pm 3.24 \mu \mathrm{M}$.

SN-38 Glucuronidation by rUGT1A1 and rUGT1A9

SN-38 is mainly converted into inactive SN38G by UGT1A1 (6) and UGT1A9 (27) in the liver. Therefore, we investigated the kinetic profiles of SN-38 glucuronidation by rUGT1A1 and rUGT1A9. Recombinant UGT1A1 or rUGT1A9 was incubated with 0-75 $\mu \mathrm{M} \mathrm{SN}-38$ for 20 min (Figure 6). SN-38 glucuronidation increased in a concentrationdependent manner up to $20 \mu \mathrm{M}$ and the increase was linear up to $5.0 \mu \mathrm{M}$. In the same way as human liver microsomes at concentrations higher than $20 \mu \mathrm{M}$, the kinetic profiles of $\mathrm{SN}-38$ glucuronidation indicated substrate inhibition (10). The enzyme kinetic parameters of $K_{\mathrm{m}}, V_{\max }, C L_{\mathrm{int}}$, and $K_{\mathrm{si}}$ by 
rUGT1A1 were $11.4 \pm 1.2 \mu \mathrm{M}, 194 \pm 13.5 \mathrm{pmol} / \mathrm{mg}$ protein $/ \mathrm{min}, 17.1 \pm 0.6 \mu \mathrm{L} / \mathrm{mg}$ protein $/ \mathrm{min}$, and 26.9 $\pm 2.2 \mu \mathrm{M}$, respectively, and those by rUGT1A9 were $12.8 \pm 2.7 \mu \mathrm{M}, 8.5 \pm 2.7 \mathrm{pmol} / \mathrm{mg}$ protein $/ \mathrm{min}, 0.68$ $\pm 0.09 \mu \mathrm{L} / \mathrm{mg}$ protein $/ \mathrm{min}$, and $23.7 \pm 16.1 \mu \mathrm{M}$, respectively.

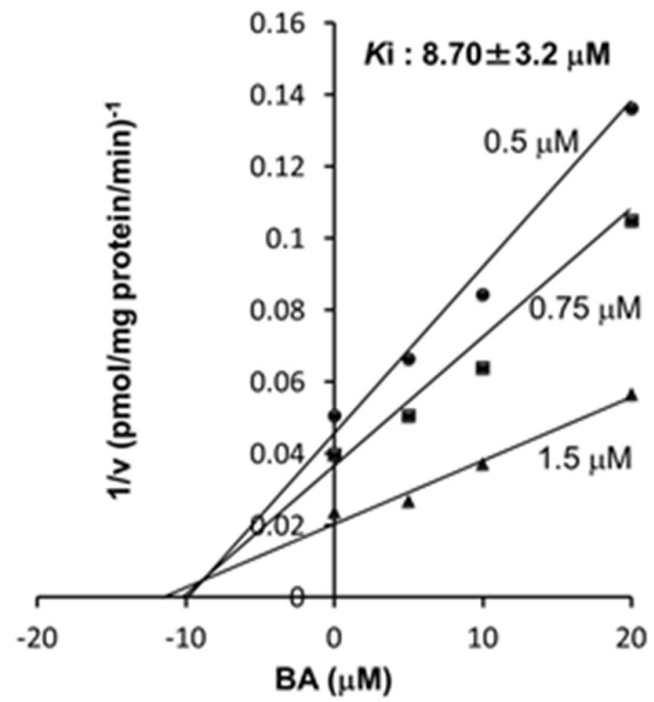

Figure 5. Dixon plots of BA on SN-38 glucuronidation in human liver microsomes. Incubation was performed for $20 \mathrm{~min}$. Each value represents the mean of triplicate determinations.

\section{Inhibitory Effect of $\mathrm{BA}$ on $\mathrm{SN}-38$ Glucuronidation by rUGT1A1}

The inhibitory effect of BA on SN-38 glucuronidation by rUGT1A1 was investigated (Figure 7). Co-incubation with BA concentrationdependently decreased SN-38 glucuronidation significantly; the addition of $5 \mu \mathrm{M}$ and $10 \mu \mathrm{M}$ BA reduced $\mathrm{SN}-38$ glucuronidation by $42 \%$ and $68 \%$, respectively. On the other hand, SN-38 glucuronidation by rUGT1A9 showed very low activity; hence, $\mathrm{SN}-38 \mathrm{G}$ could not be detected when BA was added to the incubation mixture.

\section{DISCUSSION}

The kinetic profiles of SN-38 glucuronidation in human liver microsomes were clarified. SN-38 glucuronidation increased in a time-dependent (Figure 1) and concentration-dependent manner and showed the highest activity $(250 \mathrm{pmol} / \mathrm{mg}$ protein/min) at approximately $20 \mu \mathrm{M}$ (Figure 2). However, at concentrations higher than $20 \mu \mathrm{M}$, the activity gradually decreased, indicating substrate inhibition. Some studies of SN-38 glucuronidation in human liver microsomes reported the MichaelisMenten type profile (6, 27-29), whereas $\mathrm{Wu}$ et al. (10) reported substrate inhibition profiles in human liver microsomes and rUGT1A1, which were similar to our results. Luukkanen et al. (30) pointed out that the bisubstrate (drug and UDPGA) kinetics of seven recombinant human UGTs 1A1, 1A3, 1A6, 1A7, 1A8, 1A9, and $1 \mathrm{~A} 10$ indicated substrate inhibition. However, they concluded that these substrate inhibitions did not occur under physiological conditions. We speculate that the difference in kinetic profiles among research groups is probably due to differences in incubation conditions, including the lot number of human liver microsomes, the incubation time, and the ratio of UDPGA to SN38. In particular, human liver microsomes may be affected by not only UGT contents in donor liver, but also the difference in abundance ratio of such genotypes as $\mathrm{UGT} 1 \mathrm{~A} 1 * 1$ and $\mathrm{UGT} 1 \mathrm{~A} 1 * 28$ (31). From our results, the $K_{\mathrm{si}} / K_{\mathrm{m}}$ ratio of $\mathrm{SN}-38$ glucuronidation in human liver microsomes was 5.4 (see Figure 2). Consequently, if the concentration of SN-38 in the reaction were set to a value lower than $K_{\mathrm{m}}$, a reaction system that does not undergo substrate inhibition would be realized. From the above, the concentration of SN-38 in the reaction was set to 1.5 $\mu \mathrm{M}$, which was within the linear initial reaction rate and six times lower than the $K_{\mathrm{m}}$ value.

Such glycosides as BG and GL, which contain a glucuronic acid moiety, are considered natural prodrugs in herbal medicine. Orally administered glycosides are hydrolyzed by $\beta$-glucuronidase in the intestinal microflora to form more bioactive aglycones that are absorbed and exhibit various effects. Hence, the inhibitory effects of $\beta$ glucuronidase-treated or untreated TJ-14 and TJ-114 on $\mathrm{SN}-38$ glucuronidation in human liver microsomes were examined. As shown in Figure 3, the addition of $0.05 \mathrm{w} / \mathrm{v} \%$ (final concentration) each of $\beta$-glucuronidase untreated TJ-14 and TJ-114 to the incubation mixture significantly inhibited SN-38 glucuronidation by $46 \%$ and $52 \%$ as residual activity, respectively. Katoh et al. (32) reported that when 0.1 $\mathrm{w} / \mathrm{v} \%$ each of TJ-14 and TJ-114, the amount of which was twice ours, was added to the incubation mixture, the residual activities were approximately $20 \%$ and $30 \%$, respectively. Their results coincided with ours. 

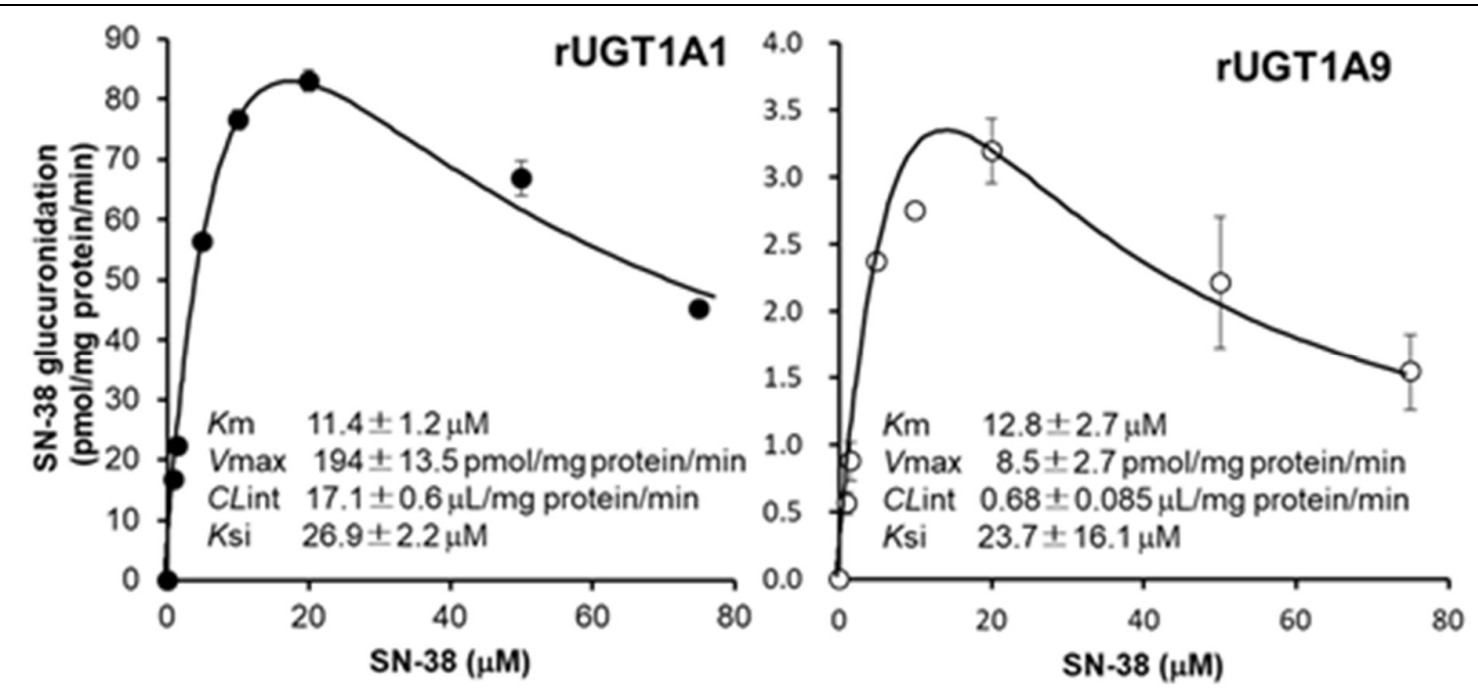

Figure 6. Kinetic profiles and parameters of SN-38 glucuronidation by rUGT1A1 and rUGT1A9. Incubation was performed for $20 \mathrm{~min}$. Each value represents the mean \pm S.D. of triplicate determinations.

We therefore suggest that $\mathrm{TJ}-14$ and $\mathrm{TJ}-114$ contain active components that inhibit SN-38 glucuronidation. However, because kampo is characterized by its complex composition, it may be difficult to demonstrate its mechanism or mode of action (20). On the assumption that $\mathrm{SN}-38 \mathrm{G}$ was hydrolyzed by $\beta$-glucuronidase from intestinal microflora and converted into the aglycone, $\beta$ glucuronidase-treated TJ-14 and TJ-114 were added to the incubation mixture. As a result, the residual activities of TJ-14 and TJ-114 were $13 \%$ and $28 \%$, respectively. Our results indicate that the inhibitory effect on SN-38 glucuronidation is enhanced in deglycosylated Japanese traditional herbal medicine. Katoh et al. (32) reported that such aglycones as BA and GA exert a greater inhibitory effect on SN-38 glucuronidation than their respective glycosides BG and GL.

The contents of the active ingredients in $5.0 \mathrm{w} / \mathrm{v} \%$ suspensions of $\beta$-glucuronidase-treated or untreated TJ-14 and TJ-114 are indicated in Table 1. These 5.0 $\mathrm{w} / \mathrm{v} \%$ suspensions corresponded to orally administered $2.5 \mathrm{~g} /$ dose (TJ-14) or $3.0 \mathrm{~g} /$ dose (TJ114) with approximately $50 \mathrm{~mL}$ of water. The contents of $\mathrm{BA}$ in $\beta$-glucuronidase-untreated TJ-14 and TJ-114 were approximately $135 \mu \mathrm{M}$ and $40 \mu \mathrm{M}$, respectively, whereas the contents of $\mathrm{BG}$ in $\beta$ glucuronidase-treated TJ-14 and TJ-114 were completely converted into $\mathrm{BA}$, resulting in approximately $2,130 \mu \mathrm{M}$ and $1,700 \mu \mathrm{M}$, respectively. On the other hand, 3MGA and GA were not detected in $\beta$-glucuronidase-untreated TJ14 and TJ-114, whereas 3MGA and GA in $\beta$ glucuronidase-treated TJ-14 and TJ-114 were slightly increased to $1-5 \mu \mathrm{M}$ and $18-37 \mu \mathrm{M}$ from GL, respectively. BG could have inhibited GL hydrolysis, and this could be the reason why GL was not completely hydrolyzed to GA by $\beta$ glucuronidase in the same way as BG hydrolysis to BA (26).

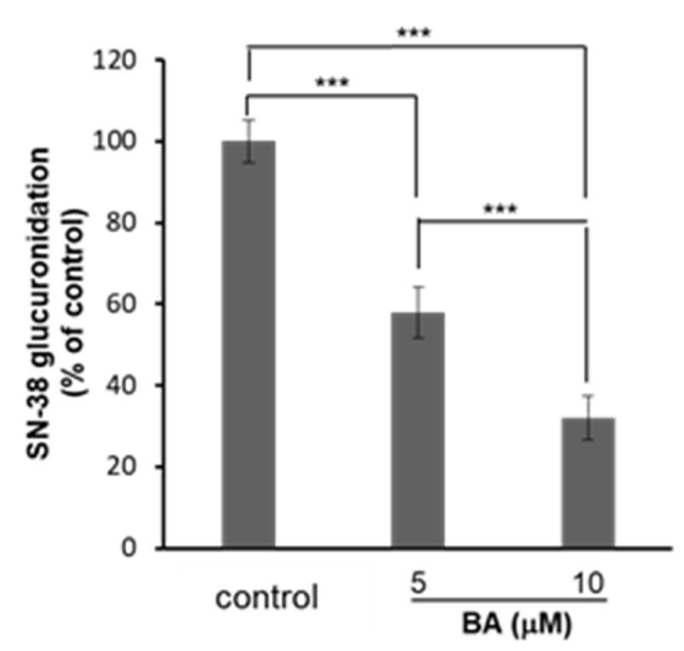

Figure 7. Inhibitory effects of BA on SN-38 glucuronidation by recombinant UGT1A1. SN-38 was set to $1.5 \mu \mathrm{M}$ and incubation was performed for $20 \mathrm{~min}$. Each value represents the mean \pm S.D. of triplicate determinations. Significantly different from control, $p<0.001$. 
The $5.0 \mathrm{w} / \mathrm{v} \%$ suspensions of TJ-14 and TJ-114 shown in Table 1 were diluted to 100 -fold their amounts by treatment with solid-phase extract and added to the reaction mixture. Therefore, the final concentrations of TJ-14 and TJ-114 in Figure 3 were $0.05 \mathrm{w} / \mathrm{v} \%$. When intestinal volume was estimated to be $500 \mathrm{~mL}$ (33), the concentrations of TJ-14 and TJ-114 were approximately $0.5 \mathrm{w} / \mathrm{v} \%$, and this value was further multiplied by 10 as the dilution coefficient. Thus, BA derived from TJ-14 and TJ114 corresponded to the addition of approximately $21 \mu \mathrm{M}$ and $17 \mu \mathrm{M}$, respectively, to the incubation mixture. Both concentrations almost agreed with 14 $\mu \mathrm{M}$, which is the $\mathrm{IC}_{50}$ of BA, as shown in Figure 4. However, conflicting results were obtained between GA contents treated with $\beta$-glucuronidase corresponding to less than $0.4 \mu \mathrm{M}$ in Table 1 and the $\mathrm{IC}_{50}$ of $15 \mu \mathrm{M}$ in Figure 4.

SN-38 glucuronidation is mainly inhibited by BA in human liver microsomes. In order to clarify the type of inhibition and the $K \mathrm{i}$ value of $\mathrm{BA}$, a Dixon plot was drawn (Figure 5). BA showed competitive inhibition and the corresponding $K \mathrm{i}$ value was $8.7 \mu \mathrm{M}$. Previous reports of human (32) and rat (34) liver microsomes yielded three times (26.9 $\mu \mathrm{M}$, mixed type) and one hundred times (970 $\mu \mathrm{M}$, mixed type) higher $\mathrm{Ki}$ values than ours. This may indicate species difference in the SN-38 glucuronidation inhibition process.

According to Hanioka et al. (35), SN-38 glucuronidation was catalyzed by UGT1A1, UGT1A3, UGT1A6, and UGT1A9 as well as by human liver microsomes. Among these UGT isoforms, UGT1A1 showed the highest activity for SN-38 glucuronidation at both low $(1 \mu \mathrm{M})$ and high $(200 \mu \mathrm{M})$ substrate concentrations. The ranking in order of activity at low and high substrate concentrations was UGT1A1 > UGT1A9 > UGT1A6 > UGT1A3 and UGT1A1 > UGT1A3 > UGT1A6 $\geq$ UGT1A9. Based on this report, the kinetic profiles of SN-38 glucuronidation catalyzed by rUGT1A1 and rUGT1A9 are shown in Figure 6. Both isoforms showed a substrate inhibition profile similar to human liver microsomes, but the $C L_{\text {int }}$ value of rUGT1A1 was approximately 25 times higher than that of rUGT1A9. These results suggest that $\mathrm{SN}-38$ glucuronidation in human liver is catalyzed primarily by UGT1A1. However, even if standardization is carried out by the negative control microsomes, each UGT expression level in the recombinant system is different from that in human liver microsomes. Therefore, care is necessary to compare with glucuronidation activities of each
rUGT isoform. This UGT1A1-catalyzed SN-38 glucuronidation was inhibited in a concentrationdependent manner by the addition of BA (Figure 7), suggesting that BA is strongly involved in the blockade of this pathway. The maximum plasma concentrations of GA and BA in humans are 0.10 $0.43 \mu \mathrm{M}(36-38)$ and $0.49 \mu \mathrm{M}$ (39), respectively. In the present study, the $K i$ value of BA in human liver microsomes was $8.7 \mu \mathrm{M}$ (Figure 5), which was higher than the plasma concentration. Although BA concentration in liver may be higher than that in plasma, it is very difficult to compare liver concentration due to lack of information.

Hirose et al. (40) reported the relationship between $\mathrm{SN}-38 \mathrm{G} / \mathrm{SN}-38$ concentration ratio and neutropenia in 70 Japanese colorectal cancer patients. The SN-38G/SN-38 concentration ratio was lower in patients who were homozygous for UGT1A $1 * 6$, heterozygous for UGT1A $1 * 6$ or UGT1A $1 * 28$, or double heterozygotes than in patients with wild-type genes. It has been proposed that BG from TJ-14 and TJ-114 inhibits bacterial $\beta$-glucuronidase (17), and the uptake of SN-38 mediated by OATP2B1 in the apical side of intestinal epithelial cells $(24,25)$, thereby reducing the risk of $\mathrm{SN}-38$-induced lateonset diarrhea. On the other hand, the deglycosylated form, BA, may raise plasma SN-38 levels by inhibiting hepatic UGT1A1 activity as well as UGT1A $1 * 6$ or $\mathrm{UGT} 1 \mathrm{~A} 1 * 28$, and consequently decrease neutrophil count. Further research is required to prove this speculation.

We summarize our findings as follows: when TJ14 and TJ-114 are orally administered, BG derived from Scutellariae radix is hydrolyzed to aglycone BA by bacterial $\beta$-glucuronidase in the intestinal tract. As the deglycosylation of GL derived from Glycyrrhizae radix is inhibited by $\mathrm{BG}$, a sufficient amount of GA could not be produced. These results led us to suggest that GA does not contribute to SN38 glucuronidation. BA is transported to liver via bloodstream. CPT-11 is hydrolyzed by carboxyesterase and converted into SN-38, and SN38 glucuronidation is mainly catalyzed by UGT1A1 in the liver. Our results strongly suggest that SN-38 glucuronidation is inhibited by BA.

\section{REFERENCES}

1. Sanghani SP, Quinney SK, Fredenburg TB, Davis WI, Murry DJ, Bosron WF. Hydrolysis of irinotecan and its oxidative metabolites, 7-ethyl-10-[4-N-(5aminopentanoic acid)-1-piperidino] carbonyloxycamptothecin and 7-ethyl-10-[4-(1- 
piperidino)-1-amino]-carbonyloxycamptothecin, by human carboxylesterases CES1A1, CES2, and a newly expressed carboxylesterase isoenzyme, CES3. Drug Metab Dispos, 2004; 32:505-511. DOI: https://doi.org/10.1124/dmd.32.5.505.

2. Rivory LP, Riou J-F, Haaz M-C, Sable S, Vuilhorgne M, Commerçon A, Pond SM, Robert J. Identification and properties of a major plasma metabolite of irinotecan (CPT-11) isolated from the plasma of patients. Cancer Res, 1996; 56:3689-3694.

3. Haaz M-C, Rivory L, Riché C, Vernillet L, Robert J. Metabolism of irinotecan (CPT-11) by human hepatic microsomes: participation of cytochrome P-450 3A and drug interactions. Cancer Res, 1998; 58:468-472.

4. Dodds HM, Haaz M-C, Riou J-F, Robert J, Rivory LP. Identification of a new metabolite of CPT-11 (irinotecan): pharmacological properties and activation to SN-38. J Pharmacol Exp Ther, 1998; 286:578-583.

5. Smith NF, Figg WD, Sparreboom A. Pharmacogenetics of irinotecan metabolism and transport: An update. Toxicol In Vitro, 2006; 20:163175. DOI: https://doi.org/10.1016/j.tiv.2005.06.045.

6. Iyer L, King CD, Whitington PF, Green MD, Roy SK, Tephly TR, Coffman BL, Ratain MJ. Genetic predisposition to the metabolism of irinotecan (CPT11). Role of uridine diphosphate glucuronosyltransferase isoform $1 \mathrm{~A} 1$ in the glucuronidation of its active metabolite ( $\mathrm{SN}-38)$ in human liver microsomes. J Clin Invest, 1998; 101:847-854. DOI: https://doi.org/10.1172/JCI915.

7. Mackenzie PI, Bock KW, Burchell B, Guillemette C, Ikushiro S, Iyanagi T, Miners JO, Owens IS, Nebert DW. Nomenclature update for the mammalian UDP glycosyltransferase (UGT) gene superfamily. Pharmacogenet Genomics, 2005; 15:677-685. DOI: https://doi.org/10.1097/01.fpc.0000173483.13689.5 6.

8. Ohno S, Nakajin S. Determination of mRNA expression of human UDP-glucuronosyltransferases and application for localization in various human tissues by real-time reverse transcriptase-polymerase chain reaction. Drug Metab Dispos, 2009; 37:32-40. DOI: https://doi.org/10.1124/dmd.108.023598.

9. Fujita K, Sparreboom A, Pharmacogenetics of irinotecan disposition and toxicity: A Review. Curr Clin Pharmacol, 2010; 5:209-217. DOI: https://doi.org/10.2174/157488410791498806.

10. $\mathrm{Wu} \mathrm{B}$, Zhang $\mathrm{S}, \mathrm{Hu} \mathrm{M}$. Evaluation of $3,3^{\prime}, 4^{\prime}-$ Trihydroxyflavone and 3,6,4'-trihydroxyflavone (4'O-glucuronidation) as the in vitro functional markers for hepatic UGT1A1. Mol Pharm, 2011; 8:2379-2389. DOI: https://doi.org/10.1021/mp200300w.
11. Sugiyama Y, Kato Y, Chu X. Multiplicity of biliary excretion mechanisms for the camptothecin derivative irinotecan (CPT-11), its metabolite SN-38, and its glucuronide: role of canalicular multispecific organic anion transporter and P-glycoprotein. Cancer Chemother Pharmacol, 1998; 42 (Suppl):S44-S49.

12. Di Paolo A, Bocci G, Danesi R, Del Tacca M. Clinical pharmacokinetics of irinotecanbased chemotherapy in colorectal cancer patients. Curr Clin Pharmacol, 2006; 1:311-323. DOI: https://doi.org/10.2174/157488406778249307.

13. Masuda N, Fukuoka M, Kusunoki Y, Matsui K, Takifuji N, Kudoh S, Negoro S, Nishioka M, Nakagawa K, Takada M. CPT-11: a new derivative of camptothecin for the treatment of refractory or relapsed small-cell lung cancer. J Clin Oncol, 1992; 10:1225-1229.

14. Kurita A, Kado S, Matsumoto T, Asakawa N, Kaneda N, Kato I, Uchida K, Onoue M, Yokokura $\mathrm{T}$. Streptomycin alleviates irinotecan-induced delayedonset diarrhea in rats by a mechanism other than inhibition of $\beta$-glucuronidase activity in intestinal lumen. Cancer Chemother Pharmacol, 2011; 67:201213. DOI: https://doi.org/10.1007/s00280-010-13104.

15. Hecht JR, Pillai M, Gollard R, Heim W, Swan F, Patel R, Dreiling L, Mo M, Malik I. A randomized, placebo-controlled phase II study evaluating the reduction of neutropenia and febrile neutropenia in patients with colorectal cancer receiving pegfilgrastim with every-2-week chemotherapy. Clin Colorectal Cancer, 2010; 9:95-101. DOI: https://doi.org/10.3816/CCC.2010.n.013.

16. Swami U, Goel S, Mani S. Therapeutic targeting of CPT-11 induced diarrhea: A case for prophylaxis. Curr Drug Targets, 2013; 14:777-797. DOI: https://doi.org/10.2174/1389450111314070007.

17. Sakata Y, Suzuki H, Kamataki T. Preventive effect of TJ-14, a kampo (Chinese herb) medicine, on diarrhea induced by irinotecan hydrochloride (CPT-11). Gan To Kagaku Ryoho, 1994; 21:1241-1244.

18. Furman WL, Crews KR, Billups C, Wu J, Gajjar AJ, Daw NC, Patrick CC, Rodriguez-Galindo C, Stewart CF, Dome JS, Panetta JC, Houghton PJ, Santana VM. Cefixime allows greater dose escalation of oral irinotecan: a phase I study in pediatric patients with refractory solid tumors. J Clin Oncol, 2006; 24:563570. DOI: https://doi.org/10.1200/JCO.2005.03.2847.

19. Narita M, Nagai E, Hagiwara H, Aburada M, Yokoi $\mathrm{T}$, Kamataki T. Inhibition of beta-glucuronidase by natural glucuronides of kampo medicines using glucuronide of $\mathrm{SN}-38$ (7-ethyl-10hydroxycamptothecin) as a substrate. Xenobiotica, 
1993; 23:5-10. DOI: https://doi.org/10.3109/00498259309059356.

20. Ikegami F, Fujii Y, Satoh T. Toxicological considerations of Kampo medicines in clinical use. Toxicol, 2004; 198:221-228. DOI: https://doi.org/10.1016/j.tox.2004.01.029.

21. Takasuna K, Kasai Y, Kitano Y, Mori K, Kobayashi R, Hagiwara T, Kakihata K, Hirohashi M, Nomura M, Nagai E, Kamataki T. Protective effects of kampo medicines and baicalin against intestinal toxicity of a new anticancer camptothecin derivative, irinotecan hydrochloride (CPT-11), in rats. Jpn J Cancer Res, 1995; 86:978-984.

22. Akao T, Distribution of enzymes involved in the metabolism of glycyrrhizin in various organs of rat. Biol Pharm Bull, 1998; 21:1036-1044. DOI: https://doi.org/10.1248/bpb.21.1036.

23. Akao T, Kawabata K, Yanagisawa E, Ishihara K, Mizuhara Y, Wakui Y, Sakashita Y, Kobashi K. Baicalin, the predominant flavone glucuronide of scutellariae radix, is absorbed from the rat gastrointestinal tract as the aglycone and restored to its original form. J Pharm Pharmacol, 2000; 52:15631568.

DOI: https://doi.org/10.1211/0022357001777621.

24. Itoh T, Itagaki S, Sumi Y, Hirano T, Takemoto I, Iseki K. Uptake of irinotecan metabolite $\mathrm{SN}-38$ by the human intestinal cell line Caco-2. Cancer Chemother Pharmacol, 2005; 55:420-424. DOI: https://doi.org/10.1007/s00280-004-0937-4.

25. Fujita D, Saito Y, Nakanishi T, Tamai I. Organic anion transporting polypeptide (OATP)2B1 contributes to gastrointestinal toxicity of anticancer drug SN-38, active metabolite of irinotecan hydrochloride. Drug Metab Dispos, 2016; 44:1-7. DOI: https://doi.org/10.1124/dmd.115.066712.

26. Miyamura M, Ono M, Kyotani S, Nishioka Y. Properties of glycyrrhizin in Kampo extracts including licorice root and changes in the blood concentration of glycyrrhetic acid after oral administration of Kampo extracts. YAKUGAKU ZASSHI, 1996; 116:209-216. DOI: https://doi.org/10.1248/yakushi1947.116.3_209.

27. Gagné JF, Montminy V, Belanger P, Journault K, Gaucher G, Guillemette C. Common human UGT1A polymorphisms and the altered metabolism of irinotecan active metabolite 7-ethyl-10hydroxycamptothecin (SN-38). Mol Pharmacol, 2002; 62:608-617. DOI: https://doi.org/10.1124/mol.62.3.608.

28. Haaz MC, Rivory L, Jantet S, Ratanasavanh D, Robert J. Glucuronidation of SN-38, the active metabolite of irinotecan, by human hepatic microsomes. Pharmacol Toxicol, 1997; 80:91-96.

DOI: $\quad$ https://doi.org/10.1111/j.16000773.1997.tb00289.x.

29. Hanioka N, Tanabe N, Jinno H, Tanaka-Kagawa T, Nagaoka K, Naito S, Koeda A, Narimatsu S. Functional characterization of human and cynomolgus monkey UDP-glucuronosyltransferase 1A1 enzymes. Life Sci, 2010; 87:261-268. DOI: https://doi.org/10.1016/j.lfs.2010.07.001.

30. Luukkanen L, Taskinen J, Kurkela M, Kostiainen R, Hirvonen J, Finel M. Kinetic characterization of the 1A subfamily of recombinant human UDPglucuronosyltrasferases. Drug Metab Dispos, 2005; 33:1017-1026.

DOI: https://doi.org/10.1124/dmd.105.004093.

31. Ando Y, Saka H, Ando M, Sawa T, Muro K, Ueoka H, Yokoyama A, Saitoh S, Shimokata K, Hasegawa Y. Polymorphisms of UDP-glucuronosyltransferase gene and irinotecan toxicity: A pharmacogenetic analysis. Cancer Res, 2000; 60: 6921-6926.

32. Katoh M, Yoshioka Y, Nakagawa N, Yokoi T. Effects of Japanese herbal medicine, Kampo, on human UGT1A1 activity. Drug Metab Pharmacokinet, 2009; 24:226-234.

DOI: https://doi.org/10.2133/dmpk.24.226.

33. Foti RS, Wahlstrom JL, Wienkers LC. The in vitro drug interaction potential of dietary supplements containing multiple herbal components. Drug Metab Dispos, 2007; 35:185-188. DOI: https://doi.org/10.1124/dmd.106.012724.

34. Yokoi T, Narita M, Nagai E, Hagiwara H, Aburada M, Kamataki T. Inhibition of UDPglucuronosyltransferase by aglycons of natural glucuronides in kampo medicines using $\mathrm{SN}-38$ as a substrate. Jpn J Cancer Res, 1995; 86:985-989.

35. Hanioka N, Ozawa S, Jinno H, Ando M, Saito Y, Sawada J. Human liver UDPglucuronosyltransferase isoforms involved in the glucuronidation of 7-ethyl-10-hydroxycamptothecin. Xenobiotica, 2001; 31:687-699. DOI: https://doi.org/10.1080/00498250110057341.

36. Zou Q, Wei P, Li J, Ge ZX, Ouyang P. Simultaneous determination of $18 \square$ - and $18 \beta$-glycyrrhetic acid in human plasma by LC-ESI-MS and its application to pharmacokinetics. Biomed Chromatogr, 2009; 23:54-62. DOI: https://doi.org/10.1002/bmc.1082.

37. Kitagawa H, Munekage M, Ichikawa K, Fukudome I, Munekage E, Takezaki Y, Matsumoto T, Igarashi Y, Hanyu H, Hanazaki K. Pharmacokinetics of active components of Yokukansan, a traditional Japanese herbal medicine after a single oral administration to healthy Japanese volunteers: A cross-over, randomized study. PLoS One, 2015; 10:e0131165. 
DOI: https://doi.org/10.1371/journal.pone.0131165.

38. Yamamura Y, Kawakami J, Santa T, Kotaki H, Uchino K, Sawada Y, Tanaka N, Iga T. Pharmacokinetic profile of glycyrrhizin in healthy volunteers by a new high-performance liquid chromatographic method. J Pharm Sci, 1992; 81:1042-1046.

DOI: https://doi.org/10.1002/jps.2600811018.

39. Li M, Shi A, Pang H, Xue W, Li Y, Cao G, Yan B, Dong F, Li K, Xiao W, He G, Du G, Hu X. Safety, tolerability, and pharmacokinetics of a single ascending dose of baicalein chewable tablets in healthy subjects. J Ethnopharmacol, 2014; 156:210215. DOI: https://doi.org/10.1016/j.jep.2014.08.031.

40. Hirose K, Yamashita K, Takada H, Kaneda N, Fukami K, Maruo E, Kitamura M, Hasegawa J, Maeda Y. Usefulness of one-point plasma SN$38 \mathrm{G} / \mathrm{SN}-38$ concentration ratios as a substitute for UGT1A1 genetic information after irinotecan administration. Int J Clin Oncol, 2014; 19:397-402. DOI: https://doi.org/10.1007/s10147-013-0558-1 
J Pharm Pharm Sci (www.cspsCanada.org) 21, 195 - 206, 2018 